\title{
Flexible Paper-Based Li-ion Batteries: A Review
}

\author{
Amirhossein Ahmadian', Abbas Shafiee ${ }^{2}$, Mohammadali Alidoost ${ }^{3}$, Amin Akbari² \\ ${ }^{1}$ Department of Mechanical and Aerospace Engineering, University of California, Los Angeles, CA, USA \\ ${ }^{2}$ School of Mechanical Engineering, Purdue University, West Lafayette, IN, USA \\ ${ }^{3}$ Department of Bioengineering, University of California, Los Angeles, CA, USA \\ Email: aahmadian@ucla.edu, shafiee@purdue.edu, aakbari@purdue.edu,malidoost@mednet.ucla.edu
}

How to cite this paper: Ahmadian, A., Shafiee, A., Alidoost, M. and Akbari, A. (2021) Flexible Paper-Based Li-ion Batteries: A Review. World Journal of Engineering and Technology, 9, 285-299.

https://doi.org/10.4236/wjet.2021.92020

Received: February 16, 2021

Accepted: May 7, 2021

Published: May 10, 2021

Copyright (c) 2021 by author(s) and Scientific Research Publishing Inc. This work is licensed under the Creative Commons Attribution International License (CC BY 4.0).

http://creativecommons.org/licenses/by/4.0/

\begin{abstract}
Lithium-ion (Li-ion) batteries have been fabricated in various ways to improve flexibility. Flexibility could be enhanced via active materials, separators, electrodes, and electrolytes, which could then be integrated to form flexible electronic devices with promising electrochemical properties compared to traditional non-flexible Li-ion batteries. Recent progress towards flexible Liion batteries fabrication, materials, and their electrochemical properties are investigated in this review. Additionally, recent developments in electronic devices utilizing flexible batteries and their future applications are explored. Portable and wearable electronics, as the primary beneficiaries of the flexible, rechargeable, and high-performance Li-ion batteries, are examined. In the end, various applications and challenges of flexible batteries in healthcare and various energy storage systems, considering practical implementation, are argued.
\end{abstract}

\section{Keywords}

Lithium-Ion (Li-Ion) Batteries, Flexible Batteries, Paper-Based Batteries

\section{Introduction}

Lithium-Ion (Li-ion) batteries have been modified in various ways to improve flexibility. One of the areas that have resulted in an extensive interest in terms of flexibility is the use of substrates made of paper for the Li-ion batteries. However, flexibility can be enhanced in various components of Li-ion batteries such as the active materials, separators, carbon nanotubes, and electrolytes, which are then integrated to form devices with better electrochemical properties [1].

The batteries, as well as other devices used in energy storage, have increasingly been made using either textile components or paper. The use of the paper-based substrate has been on the rise due to various advantages, it has over other mate- 
rials. While flexibility is an important feature or characteristic of the material, its intrinsic nature also provides other advantages. The substrate can offer outstanding or high performance while at the same time saving on cost and supporting multi-functionality [2].

The paper-based material also exhibits higher versatility and more effective disposability than silicon or glass, which are conventionally inflexible materials. Apart from being ubiquitously accessible and combustible, the paper is also economically disposable, biodegradable, and biocompatible [3]. These properties are exhibited by the paper substrate since its main constituent is cellulose, which offers desirable qualities in terms of porosity and flexibility. Combining it with carbon nanotubes further improves the characteristics.

Additionally, the paper also provides a large surface area for the storage of reagents while it also has the ability to wick liquids through the action of capillarity [4].

In this review, recent flexible batteries fabrication, materials, and their properties are explored. The difference between a typical Li-ion cell versus a flexible paper-based cell is investigated. Recent research findings on flexible paper-based substrates in Li-ion batteries to improve their applications in high energy and power requirements are reported. Various recent flexible batteries based on their electrochemical performance, mechanical and material properties, flexibility are compared and investigated.

In the end, the main beneficiaries of the flexible Li-ion batteries in the healthcare industry, wearable electronics, and renewable energy systems are discussed, and their future prospect due to their capacity to generate high power and densities of energy while offering a guarantee of a long lifespan are explored.

\section{Flexible Batteries Fabrication, Materials, Electrochemical and Mechanical Properties}

\subsection{Rigid Li-Ion Cells versus Flexible Paper-Based Cells}

Li-ion batteries manufacturing has adopted the new developments and advancements made in the use of paper substrates. The use of paper in the manufacturing of flexible Li-ion batteries began with the exploration of the material as a feasible substrate in devices used to store energy, which showed its excellent conductivity, porosity, and stability in both mechanical and chemical perspectives [4] [5] [6]. However, technological advancements in this area have also witnessed various challenges.

The difference between a typical Li-ion cell versus a flexible paper-based cell consists of a cellulose-bound graphite anode, cellulose-bound $\mathrm{LiFePO}_{4}$ cathode, and a standard glass-wool separator soaked in a liquid electrolyte is depicted as following (see Figure 1).

Li-ion battery has been one of the primary devices that have benefitted from the developments in flexible and portable electronics. A simple and flexible Li-ion battery can be fabricated on a nanocomposite substrate paper that has a cathode 


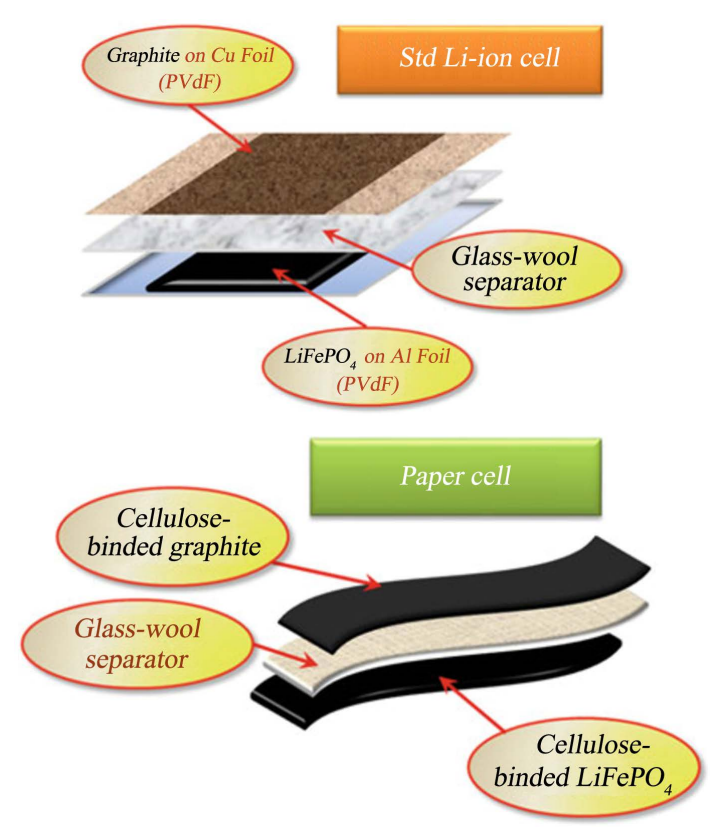

Figure 1. A standard Li-ion cell (upper image) versus a flexible paper-based cell consist of a cellulose-binded graphite anode + cellulose-binded $\mathrm{LiFePO}_{4}$ cathode + glass-wool separator soaked in a liquid electrolyte (lower image) [6].

and anode made of a Li layer of metal [3]. Numerous explorations have been carried out to use flexible paper-based substrates in Li-ion batteries to improve their applications in high energy and power requirements and replace $\mathrm{Ni}-\mathrm{Cd}$ or $\mathrm{Ni}-\mathrm{MH}$ types. The interest in flexible electronics has led to various research in the use of paper, textile, and polymer substrate in Li-ion batteries. The porous nature of these materials has enhanced the research studies since it enhances the generation of power [7].

\subsection{Fabrication, Material, Electrochemical and Mechanical Properties}

One of the studies was conducted by developing a Li-ion battery of single-walled carbon nanotube/poly-cellulose paper (SWCNT/PP). Using $\mathrm{LiFePO}_{4}$ and $\mathrm{Li}_{4} \mathrm{Ti}_{5} \mathrm{O}_{1}$ electrodes, the full cells based on the SWCNT/PP exhibited a rapid capacity of discharge of about $153.3 \mathrm{~mA} \cdot \mathrm{hg}^{-1}$ with $90.6 \%$ of columbic efficiency at $0.1^{\circ} \mathrm{C}$ [2] (see Figure 2). Using special cellulose of nano-fibrillated type that served as electrode binder and material for separation, the manufacture of the battery was done by paper-making process. The resulting Li-ion batteries were established to have strong mechanical properties, flexibility, excellent cycling properties.

Flexible Li-ion batteries have also been made on the basis of the paper-based hierarchical $\mathrm{TiO}_{2}$ of film electrode where the paper filters were considered as the skeletons for the formation of the film. The application of the $\mathrm{TiO}_{2}$ film in the Li-ion batteries showed that the performance rate increased while the capacity remained relatively constant at about $100 \mathrm{~mA} \cdot \mathrm{hg}^{-1}$ even when the rates rose to $20^{\circ} \mathrm{C}$. However, research in this area is complicated by the complex processes, 


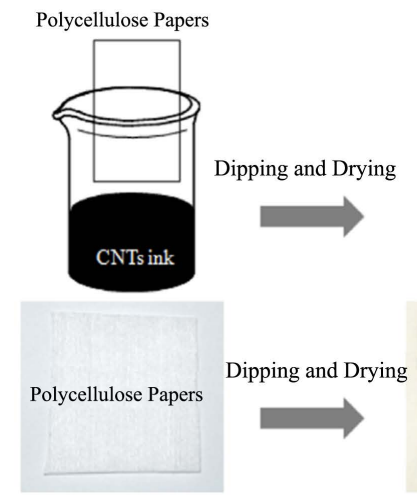

(a)

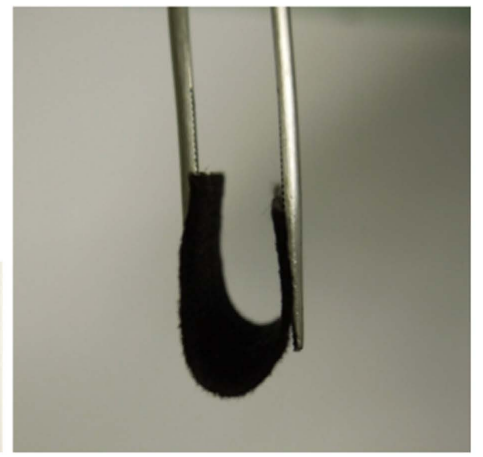

(b)

Figure 2. (a) Schematic SWCNT/PPs fabrication process. (b) SWCNT/PPs showing flexibility behavior [2].

which also increase the associated costs. The costs can be decreased by reducing complexity, such as using readily available paper for fabrications. The paper can be coated with materials such as carbon nanotube [8] [9] (see Figure 3).

In Figure 4, a schematic of the flexible Li-ion paper battery (paper as both the separator and the mechanical support can be seen lighting up a LED device.

Generally, the paper-based substrate enhanced film adhesion compared to other materials such as plastics. It also streamlined or simplified the process of coating and reduced the costs. The study indicated that the conductive paper could be applied as a current collector with considerable success, in which case they can replace the metallic types with estimated capacitance retention of $95 \%$ within 280 cycles [2]. As well as exhibiting good cycling performance, the Li-ion batteries managed to register $0.01 \%$ capacity decay within 220 cycles. It can be established that flexible paper-based Li-ion batteries have much better power sources in relation to the generation of both current and power compared to electrochemical and biofuel batteries.

Additionally, the Li-ion paper-based batteries have the ability to keep a charge for more extended periods. However, one of the drawbacks of these flexible Li-ion batteries is the need to have several functional layers that are deposited on the paper, which means design and fabrication complexities. Electrode degradation due to external loading, inefficient performance, and the complicated procedure of preparing the cells are also challenges faced in using the flexible Li-ion batteries [10]. Additionally, integrating with other electronics makes it necessary to have excellent packaging and insulation techniques.

Flexible Li-ion batteries also employ paper-folding techniques to compact the battery and enhance its energy in every footprint surface area. The art used in paper-folding has been used frequently to increase compactness in Li-ion batteries while at the same time offer other benefits. Since paper is used as a substrate in these batteries, the paper-folding techniques have been sufficiently exploited in their manufacturing. The application of the techniques is important because it offers a high density of real energy using conventional materials. 


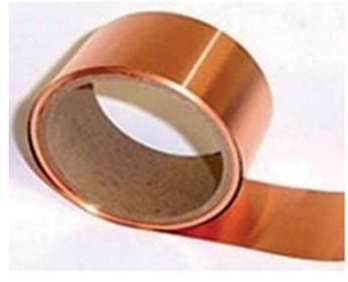

(a)

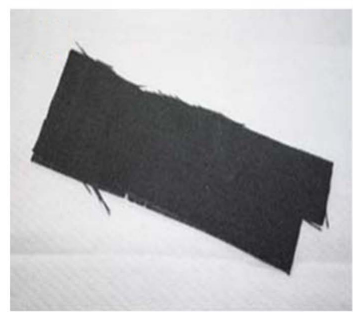

(c)

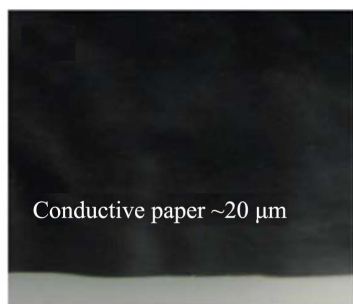

(b)

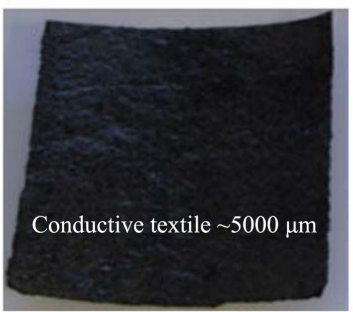

(d)

Figure 3. (a) Heavy metal foil, i.e. Cu foil traditional conductors (b), ((c), (d) thin conductors, conductive paper to replace traditional conductors based on paper and textiles [8].

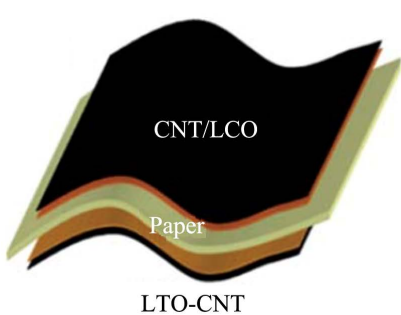

(a)

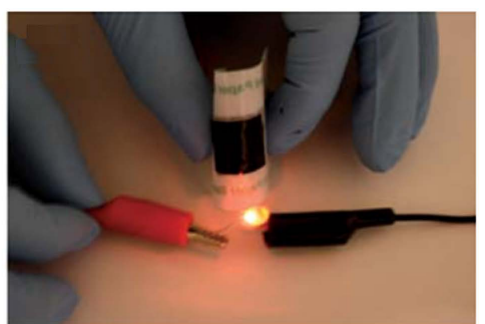

(b)

Figure 4. (a) Schematic of the flexible Li-ion paper battery (paper as both the separator and the mechanical support) (b) Picture of a flexible Li-ion battery lighting up a LED device [8].

$\mathrm{Li}$-ion batteries have been found to benefit from the techniques, including the simple methods and the complex ones such as Miura folding [11] (see Figure 5). A research study used $\mathrm{Li}$-ion batteries made from CNT papers to ensure the coated areas were conductive. Scanning electron microscopy (SEM) was used in the generation of images, which showed rope formation of the CNTs that were uniformly distributed on the paper surface. Cheng et al. made important findings in relation to the flexibility of Li-ion batteries using paper substrates.

The loss due to irreversible capacity was attributed to the interphase creation at the solid electrolyte or other reactions like those with functional groups on the carbon nanotubes, which were unavailable after the initial charge. The experiment also assessed the impact of paper-folding on the electrodes' characteristics, with copper and aluminum foils being used to provide extra current collectors. With different cell geometrical arrangements, it was established that the folded cells exhibited similar voltage profiles to those of the unfolded and planar cells. Additionally, they showed the same gravimetric capacities. A further examination of the charge and discharge curves for both unfolded and folded cells 

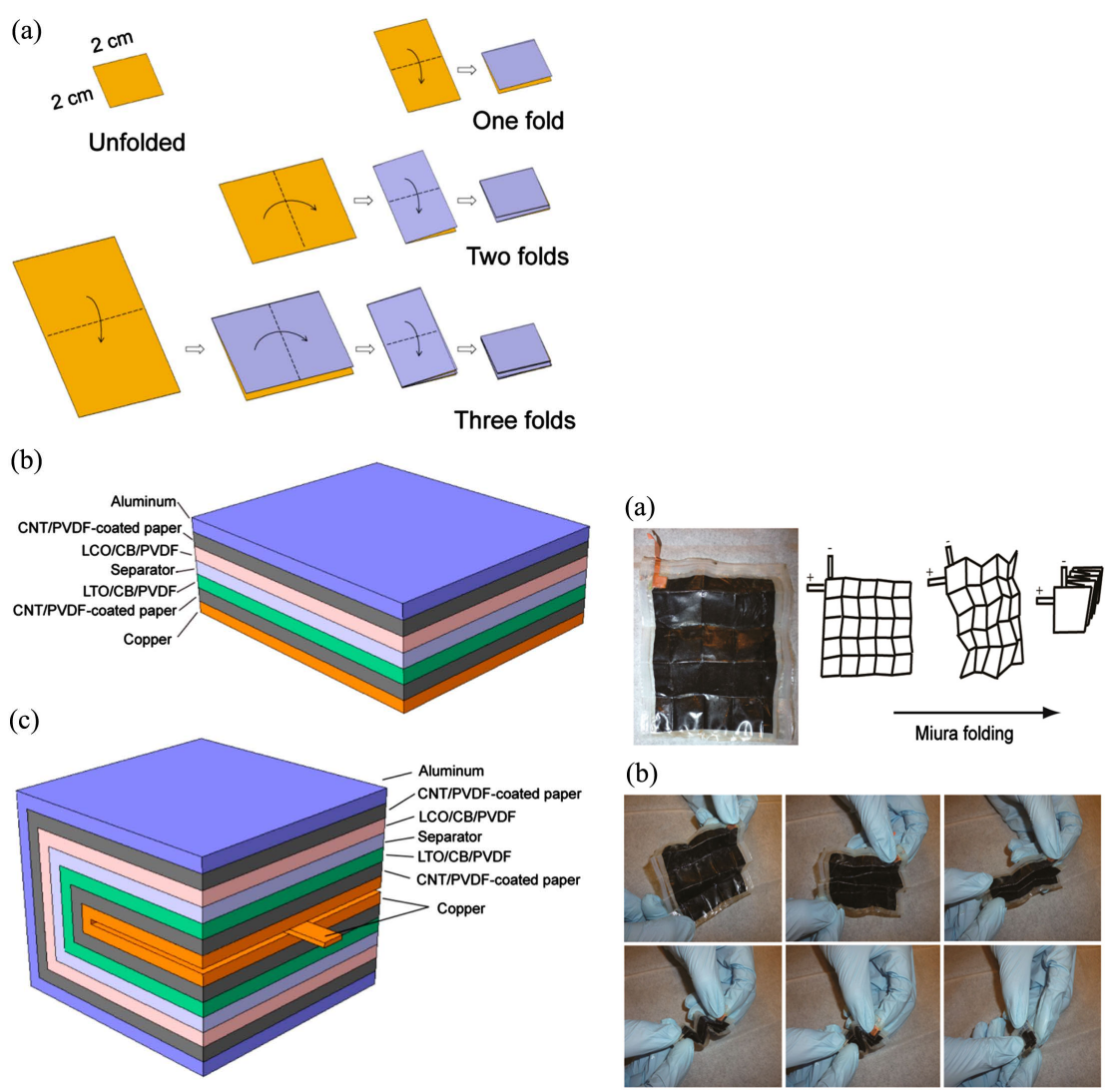

Figure 5. Left: (a) Schematic of folding procedures for batteries with one fold, two folds, and three folds. (b) Schematic showing planar, unfolded full cell, and (c) full cell with one fold. Right: (a) Schematic of Miura folding procedures sealed in Parylene-C (unfolded state). (b) Photographs of Miura folding to compact the battery to its folded state [11].

showed that the Li-ion batteries' real capacity increased relatively with the increase in the number of folds due to the increase in the active surface area [11].

The folded cells' columbic efficiency was found to be higher for the folded than the unfolded cells, which was attributed to the contact improvement between CNTs and the active components after the folding process. The flexibility, high conductivity, and electrochemical performance of Li-ion batteries are thus maintained even after multiple folding [12].

It has also been discerned that flexible Li-ion batteries did not exhibit significant delamination, cracking, or microstructure alteration, which was prominent in the planar electrodes. The new flexible shapes of the Li-ion battery have enabled manufacturers to solve problems in planar structures related to bulkiness and rigidity [13]. Therefore, new developments can be said to have improved adaptability, flexibility, miniaturization, and weave-ability.

The importance of flexible Li-ion batteries in energy storage has increased the interest in the area, especially with the growing application of such devices in modern systems. Some modern applications with vastly applied thin, flexible devices include sensing using radio frequency, interactive packaging, and other consumer products [14]. 
Recently, the Li-ion batteries have employed the same technology where the paper is used as a separator while the current collectors are implemented using CNT films. Through the process of lamination, the freestanding CNT films and other materials of the Li-ion battery are integrated on one paper sheet, which is used not only as of the separator membrane but also as a mechanical substrate. The use of the current collectors for the cathode and anode is justified by its desirable qualities, such as low film resistance of about $5 \mathrm{ohm} / \mathrm{sq}$, the lightweight of about $0.2 \mathrm{mg} / \mathrm{cm}^{2}$, and better flexibility. Even after packaging, the Li-ion batteries, which are also rechargeable, still show robust flexibility by the ability to be bent downwards up to $6 \mathrm{~mm}$ with a thickness of just $300 \mu \mathrm{m}$ [15] (see Figure 6).

According to $\mathrm{Hu}$ et al., 2010, when all Li-ion battery components are laminated into a single sheet, the intrinsic porous nature of the paper is used as a low impedance separator while also offering excellent cyclability, which ensures there is a degradation of the battery event after recharging in 300 cycles. The CNT films can be coated with stainless steel (SS) in a solution or aqueous process consisting of $10 \%$ sodium dodecylbenzene sulfonate (SDBS), which is the surfactant [15]. They suggest that there is a weak interaction between CNT films and metal substrates, which enables the manufacturing process to fabricate the films with the electrodes of the battery and the current collector. The double-layered film offers good mechanical integrity and the desired flexibility.

(1)
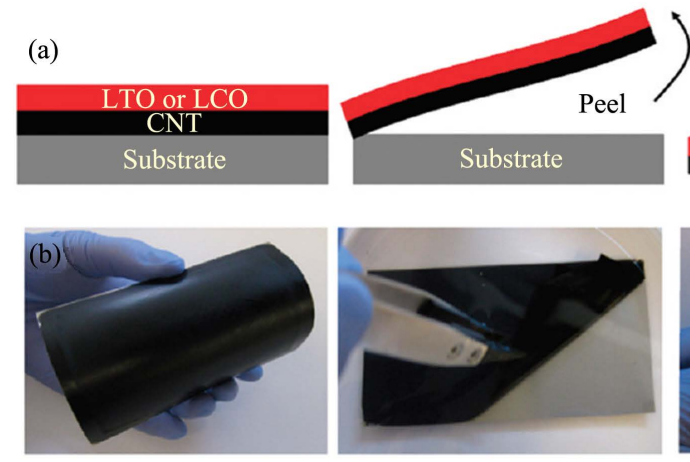

(c)

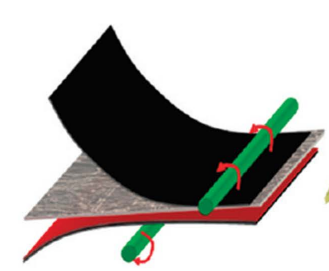

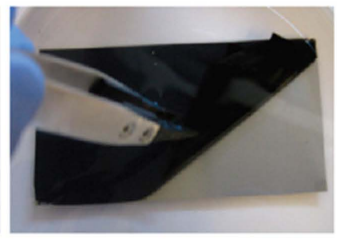

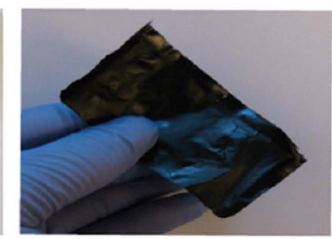

(d)

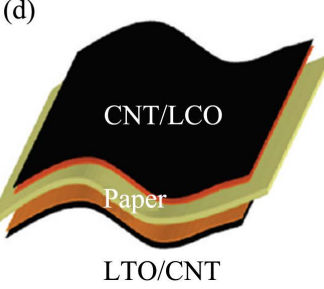

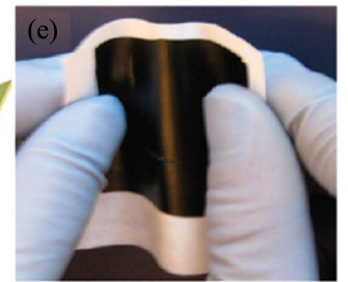

Figure 6. (a) Schematic of fabrication process of free-standing LCO/CNT: The CNT film is doctor-bladed onto the SS substrate and dried. Next, an LTO or LTO slurry is doctor-blade-coated on top of CNT film and dried. Then, they are immersed into DI water, and LCO/CNT can be peeled off (b) (Left) LTO/CNT double layer film coated on SS substrate; (middle) the double layer film can be easily peeled off from the SS substrate in DI water; (right) the final free-standing film after drying. (c) Schematic of the lamination process with a rod and a thin layer of wet PVDF on paper. (d) Schematic of the final paper Li-ion battery device [15]. 
The use of paper-like membrane or substrate as a separator in various energy storage devices has always been done, for example, in supercapacitors. However, the same technology has been adopted for Li-ion batteries, where the paper is used in separation as well as mechanical support. Large fibers and roughness are visible on the Xerox paper surface under SEM examination. The peak to valley roughness is estimated at about $10 \mu \mathrm{m}$ and while the fibers are about $20 \mu \mathrm{m}$ in diameter [4].

The lack of micro-sized holes on the paper surface gives it an excellent feature for use as a Li-ion separator. The importance of lamination cannot be underestimated in the whole process. It provides a practical approach or way of addressing leakage problems associated with the use of Xerox paper as the separator. This can be demonstrated by coating the flexible Li-ion battery's electrodes with similar slurries that are placed directly on any side of the paper. It is found that there is occasional leakage problem of electrode materials leading to device shorting through the Xerox paper. Therefore, the lamination process is essential in avoiding leakage issues since there is a form of a solid film that is integrated with the current collector, which is visible through SEM. The morphology is continuous with a considerable thickness of $2 \mu \mathrm{m}$, which prevents leakage into the porous surface of the $100 \mu \mathrm{m}$ thick Xerox paper [4] (see Figure 7).
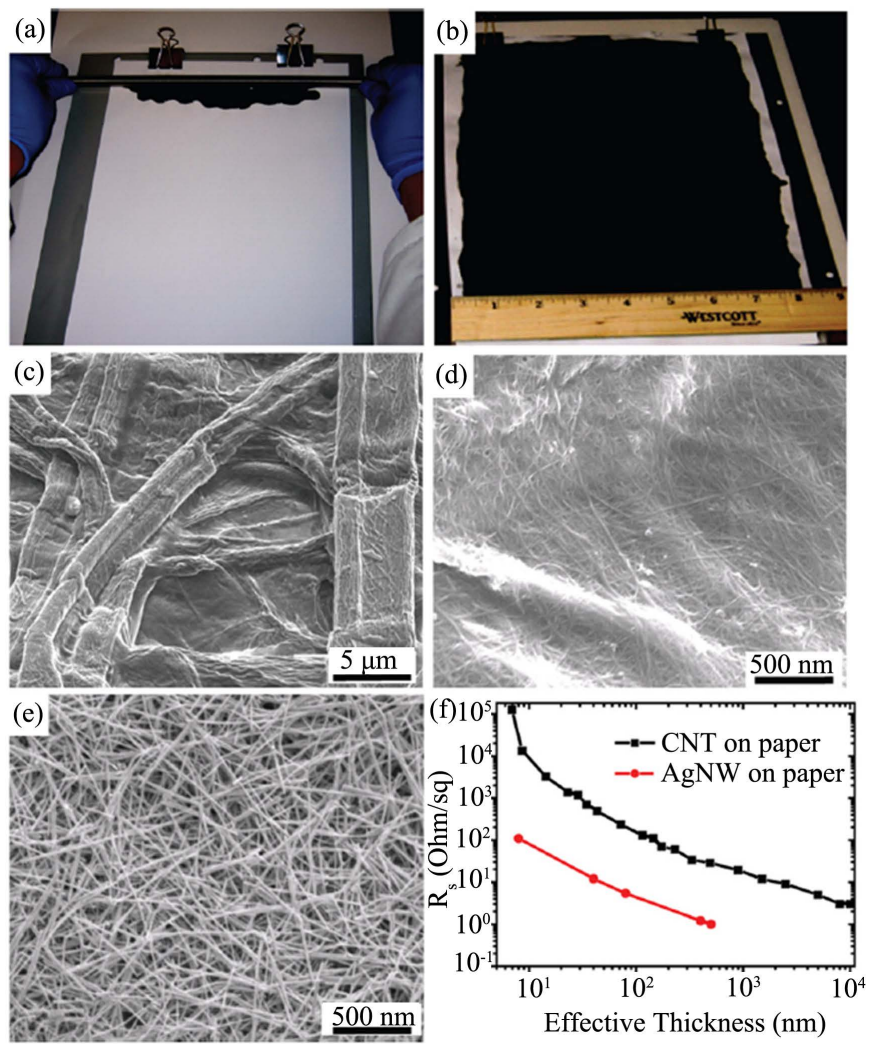

Figure 7. Coating of CNTs or Ag NWs on Xerox paper. (a) and (b) Coating of CNT or Ag NW ink on commercial Xerox paper. (c) SEM images of Xerox paper, (d) CNT coating along fibers on Xerox paper, and (e) Ag NW coating on Xerox paper. (f) Sheet resistances of conductive paper based on CNTs and Ag NWs vs. effective thicknesses [4]. 
The performance of the thin paper for flexible Li-ion batteries can be easily evaluated. Its effectiveness in performance relates to its function as a separator, stability within an electrolyte, and its effect on the impurities. These are mainly done in $\mathrm{OH}$ groups and under large ranges of voltage with respect to the lithium element being tested. Cycling the cells with different current densities, it is evident that charging and discharging capacities are minimal, thus making the irreversible capacities originating from the current collector and the separator negligible [15]. The excellent performance of paper is also underlined in its low resistivity when it interacts with the electrolyte. Generally, the self-standing paper substrate, which is flexible, has been proven to offer unprecedented levels of design ingenuity, which not only saves on costs but also results in devices with the required shape and size depending on the application [3].

Additionally, the feasibility of the Xerox paper material as a separator can be determined through certain experiments. These involve making half-cells from CNT/LTO with Li foil or CNT/LCO with the same film as the countering electrode. The experiment reveals that there is a close match between the voltage profiles of the demonstration with those that use metallic collectors of current. It also revealed that there was no apparent drop in the voltage levels for cycles occurring at the first, thirtieth, and three hundredth instances. CNTs are also crucial in the manufacturing process of flexible $\mathrm{Li}$-ion batteries.

\section{Electronic Devices Using Flexible Li-Ion Batteries and Applications}

\subsection{Various Applications and Challenges}

The world of portable electronics is the primary beneficiary of the flexible, rechargeable, and high-performance Li-ion batteries primarily due to their capacity to generate high power and densities of energy while offering a guarantee of a long lifespan [5].

The main challenge facing the use of aligned CNTs in Li-ion batteries is the absence of an excellent electrical connection between the tubes, a problem that has had limited success in terms of offering solutions [16]. In fact, even the deposition of metals at the top of the tubes to enhance electrical connectivity has not achieved the desired results. However, flexible Li-ion batteries are among the most important power sources that are applied in wearable electronics that need safe and miniaturized sources of energy [17]. They are also a useful technology in the Internet of Everything (IOE) systems, hybrid or fully electric automobiles, and power tools, which have similar requirements of long life and high density of energy [18]. If, for example, the flexible Li-ion batteries were to be widely used in electric vehicles, it would expressively decrease the amount of greenhouse emission of gases. They can also be the right choice in harvesting different renewable energy types such as solar, wind, and geothermal [19]. Therefore, the use of flexible Li-ion batteries is likely to increase interest among various government agencies. 


\subsection{Flexible Li-Ion Batteries in Healthcare and Wearable Electronics}

Technology can be considered a driving force behind improvements in healthcare. Flexible thin-film lithium-ion batteries play a key role in smart interfacing between various biological organs such as skin, heart, brain, etc., with electronicmedical (both wearable and implantable) devices. Recent advances in the design of orthodontic devices let orthodontists treat patients more safely and efficiently. One of the most common concern [1] among patients who need orthodontic treatment is the duration of the treatment but smart dental braces by taking near-infrared light therapy can enhance bone regeneration which is essential for tooth movement in orthodontic and reduces the time period and overall costs as it relies on photo-bio-modulator and osteogenic cell stimulator [20]. This orthodontic architecture requires convenient and flexible humanmachine interfaces to the human dental arch curvature $(>10 \mathrm{~mm})$. A flexible thin-film lithium-ion battery can be thinned down to achieve flexibility [21] (see Figure 8).

The smart lens system is a new wearable electronics platform with numerous applications in visual-guide or real-time health-monitoring systems. However, there have always been challenges in developing this technology due to the inadequate development of flexible batteries. Li et al., designed and applied all-solid-state thin-film batteries on polyimide substrate-based contact lens which was formed through low-temperature annealing processes [22] [23].

Wearable and portable electronics have attracted considerable attention in recent years. Although flexible and bendable batteries to power them are rapidly growing, providing sufficient energy to power wearables is challenging, especially in medical applications [25] [26] [27] [28], where continuous monitoring is required. Over the past three decades, Li-ion batteries (LIBs) have become a reliable choice for powering most electronic devices due to the ability to fabricate them with high flexibility [23].

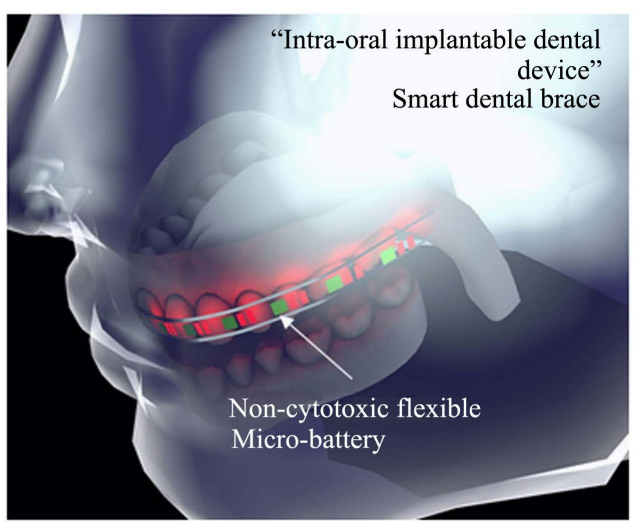

(a)

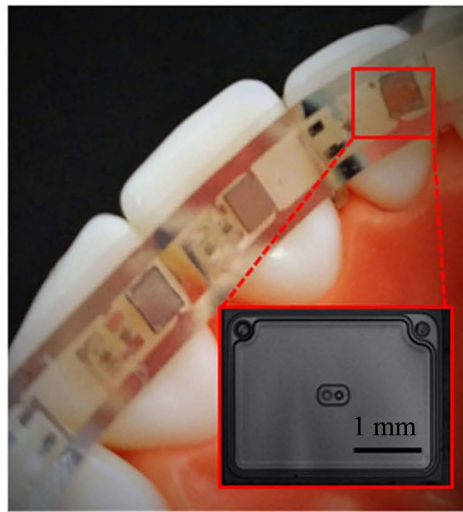

(b)

Figure 8. (a) Flexible, non-cytotoxic battery concept and integrated intra-oral phototherapeutic device fits in onto the human dental arch. (b) millimeter-sized flexible, biocompatible lithium-ion battery as a powering solution [19]. 
Zhao et al. demonstrated a wearable smart watch for continuous monitoring of sweat glucose levels by utilizing flexible photovoltaic cells for energy harvesting/ conversion and flexible $\mathrm{Zn}-\mathrm{MnO}_{2}$ batteries as energy storage. This ability to fabricate flexible power sources on this smart watch makes it a desirable device in terms of size, safety, and user comfort [24] (see Figure 9).

Textiles are types of cloth or fabric, specifically those formed by weaving, knitting, crocheting, etc. These materials can also create typically manufactured goods, such as blankets, accessories, gloves, hats, or plastic, industrial, or medical fabric. Most of the textiles exhibit high flexibility and can quickly return to their original shape after being bent or wrinkled. Nowadays, fabric batteries are inspired by the textile's fabrication techniques to integrate the $1 \mathrm{D}$ battery into a flexible textile matrix. For instance, Peng's group fabricated flexible coaxial fiber LIB and subsequently woven it into a flexible textile (Figure 8(b)) that exhibits flexibility against the huge deformations from stretching, bending, and folding. Additionally, fabric batteries provide a long working time due to the increased energy storage capacity that indicates a promising future for the next generation of wearable electronic devices and smart fabrics [25] (see Figure 10).

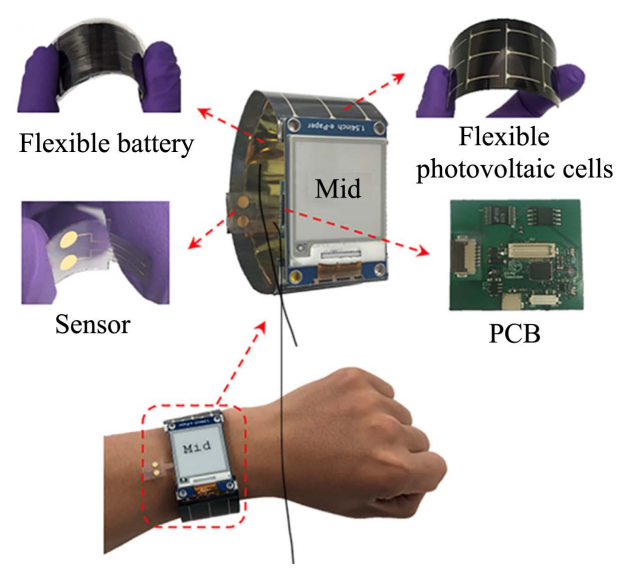

Figure 9. Pictures of a the smart watch on a wrsit with different components [24].

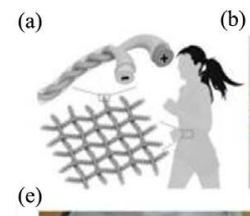

(b)

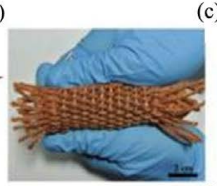

(c)

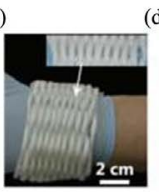

(d)
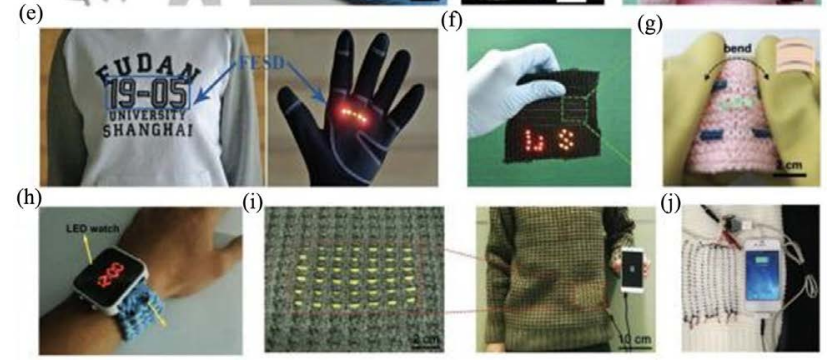

Figure 10. (a) Schematic of 1-D batteries applied in wearable applications. ((b)-(d)) 1-D batteries woven into various flexible textiles. ((e)-(h)) Watch and LED light powered by wearable textile batteries. (i), (j)) Cell-phone charged by wearable textile batteries [25]. 


\section{Summary and Future Prospects}

Over the past three decades, Li-ion batteries (LIBs) have become a reliable choice for powering most electronic devices due to the ability to fabricate them with high flexibility

The current technologies in paper-based or paper-like batteries and energy storage devices have been summarized in this review. Recent flexible batteries fabrication, materials, and their properties were investigated. Various recent flexible batteries based on their electrochemical performance, mechanical and material properties, flexibility were explored.

The primary applications of the flexible Li-ion batteries in the healthcare industry, wearable electronics, and their challenges were discussed, and their prospects, due to their capacity to generate high power and densities of energy while offering a guarantee of a long lifespan were explored.

The successful performance of paper-based electronics in flexible devices has been explored for the development of flexible, self-powered systems based on different fabrication methods and material selection. The choice of battery type depends on the application. Small power paper-based sensors might require electrochemical batteries or biofuel cells as energy sources, while other high-power electronics would prefer Lithium-ion batteries or supercapacitors. Since paper Li-ion batteries, supercapacitors are solid-state flexible power sources exploiting paper's porous, flexible and stretchable characteristics, the use of paper for these applications is limited to forming high-performance conductive paper-like substrates combining nanocomposites. Therefore, their applications are preferable as a flexible power source integrated with non-paper flexible electronics [29] [30].

Wearable and portable electronics have gained considerable attention in recent years. Although flexible and bendable batteries to power them are continuously growing, providing sufficient energy to power wearables is challenging, especially in medical applications, where continuous monitoring is required [31]-[36].

In conclusion, Lithium-ion (Li-ion) batteries have been fabricated in various ways to improve flexibility. Flexibility could be enhanced via active materials, separators, electrodes, and electrolytes, which could then be integrated to form flexible electronic devices with promising electrochemical properties compared to traditional non-flexible Li-ion batteries. Li-ion battery manufacturing has adopted new developments and advancements through the use of paper substrates. The use of cellulose paper in the manufacturing of flexible Li-ion batteries began with exploring the material as a feasible substrate in devices used to store energy, which shows its excellent conductivity, porosity, and stability in both mechanical and chemical perspectives. However, technological advancements and mass production of flexible Li-ion batteries remain a challenge for future research endeavors. 


\section{Conflicts of Interest}

The authors declare no conflicts of interest regarding the publication of this paper.

\section{References}

[1] Gwon, H., Hong, J., Kim, H., Seo, D.-H., Jeon, S. and Kang, K. (2014) Recent Progress on Flexible Lithium Rechargeable Batteries. Energy \& Environmental Science, 7, 538-551. https://doi.org/10.1039/C3EE42927I

[2] Nguyen, T.H., Fraiwan, A. and Choi, S. (2014) Paper-Based Batteries: A Review. Biosensors and Bioelectronics, 54, 640-649. https://doi.org/10.1016/j.bios.2013.11.007

[3] Pushparaj, V.L., Shaijumon, M.M., Kumar, A., Murugesan, S., Ci, L., Vajtai, R., et al. (2007) Flexible Energy Storage Devices Based on Nanocomposite Paper. Proceedings of the National Academy of Sciences of the United States of America, 104, 13574-13577. https://doi.org/10.1073/pnas.0706508104 https://www.ncbi.nlm.nih.gov/pmc/articles/PMC1959422/pdf/zpq13574.pdf

[4] Hu, L., Choi, J.W., Yang, Y., Jeong, S., La Mantia, F., Cui, L.-F., et al. (2009) Highly Conductive Paper for Energy-Storage Devices. Proceedings of the National Academy of Sciences of the United States of America, 106, 21490-21494. https://doi.org/10.1073/pnas.0908858106 https://www.ncbi.nlm.nih.gov/pmc/articles/PMC2799859/pdf/zpq21490.pdf

[5] Fu, K.K., Cheng, J., Li, T. and Hu, L. (2016) Flexible Batteries: From Mechanics to Devices. ACS Energy Letters, 1, 1065-1079. https://doi.org/10.1021/acsenergylett.6b00401

[6] Zolin, L. (2017) Large-Scale Production of Paper-Based Li-Ion Cells. Springer International Publishing, Berlin.

[7] Ahmadian, A. (2019) Design and Fabrication of High Capacity Lithium-Ion Batteries Using Electro-Spun Graphene Modified Vanadium Pentoxide Cathodes. Purdue University Graduate School, West Lafayette.

[8] Hu, L. and Cui, Y. (2012) Energy and Environmental Nanotechnology in Conductive Paper and Textiles. Energy \& Environmental Science, 5, 6423-6435. https://doi.org/10.1039/c2ee02414d

[9] Nyholm, L., Nyström, G., Mihranyan, A. and Strømme, M. (2011) Toward Flexible Polymer and Paper-Based Energy Storage Devices. Advanced Materials, 23, 3751 3769. https://doi.org/10.1002/adma.201004134

[10] Cha, H., Kim, J., Lee, Y., Cho. J. and Park, M. (2017) Issues and Challenges Facing Flexible Lithium-Ion Batteries for Practical Application. Small, 14, Article ID: e170 2989. https://doi.org/10.1002/smll.201702989

[11] Cheng, Q., Song, Z., Ma, T., Smith, B.B., Tang, R., Yu, H., et al. (2013) Folding Paper-Based Lithium-Ion Batteries for Higher Areal Energy Densities. Nano Letters, 13, 4969-4974. https://doi.org/10.1021/nl4030374

[12] Qu, H., Hou, J., Tang, Y., Semenikhin, O. and Skorobogatiy, M.J. (2016) Thin Flexible Lithium-Ion Battery Featuring Graphite Paper Based Current Collectors with Enhanced Conductivity. Canadian Journal of Chemistry, 95, 169-173. https://doi.org/10.1139/cjc-2015-0593

[13] Hoshide, T., Zheng, Y., Hou, J., Wang, Z., Li, Q., Zhao, Z., et al. (2017) Flexible Lithium-Ion Fiber Battery by the Regular Stacking of Two-Dimensional Titanium Oxide Nanosheets Hybridized with Reduced Graphene Oxide. Nano Letters, 17, 
3543-3549. https://doi.org/10.1021/acs.nanolett.7b00623

[14] Leijonmarck, S., Cornell, A., Lindbergh, G. and Wågberg, L.J. (2013) Single-Paper Flexible Li-Ion Battery Cells through a Paper-Making Process Based on Nano-Fibrillated Cellulose. Journal of Materials Chemistry A, 1, 4671-4677. https://doi.org/10.1039/c3ta01532g

[15] Hu, L., Wu, H., La Mantia, F., Yang, Y. and Cui, Y. (2010) Thin, Flexible Secondary Li-Ion Paper Batteries. ACS Nano, 4, 5843-5848. https://doi.org/10.1021/nn1018158

[16] Chen, J., Liu, Y., Minett, A. I., Lynam, C., Wang, J. and Wallace, G.G. (2007) Flexible, Aligned Carbon Nanotube/Conducting Polymer Electrodes for a Lithium-Ion Battery. Chemistry of Materials, 19, 3595-3597. https://doi.org/10.1021/cm070991g

[17] Kutbee, A.T., Ghoneim, M.T., Ahmad, S.M. and Hussain, M.M. (2016) Free-Form Flexible Lithiumion Microbattery. IEEE Transactions on Nanotechnology, 15, 402408. https://doi.org/10.1109/TNANO.2016.2537338

[18] Nitta, N., Wu, F., Lee, J.T. and Yushin, G.J.M. (2015) Li-Ion Battery Materials: Present and Future. Materialstoday, 18, 252-264.

https://doi.org/10.1016/j.mattod.2014.10.040

[19] El Baradai, O. (2014) Elaboration of Flexible Lithium-Ion Electrodes by Printing Process. Thesis, Grenoble University.

[20] Jiang, F., Roberts, W.E., Liu, Y., Shafiee, A. and Chen, J. (2020) Mechanical Environment for Lower Canine T-Loop Retraction Compared to En-Masse Space Closure with a Power-Arm Attached to Either the Canine Bracket or the Archwire. The Angle Orthodontist, 90, 801-810. https://doi.org/10.2319/050120-377.1

[21] Kutbee, A.T., Bahabry, R.R., Alamoudi, K.O., Ghoneim, M.T., Cordero, M.D., Almuslem, A.S., et al. (2017) Flexible and Biocompatible High-Performance solid-State Micro-Battery for Implantable Orthodontic System. npj Flexible Electronics, 1, Article No. 7. https://doi.org/10.1038/s41528-017-0008-7

[22] Lee, H., Kim, S., Kim, K.-B. and Choi, J.-W. (2018) Scalable Fabrication of Flexible Thin-Film Batteries for Smart Lens Applications. Nano Energy, 53, 225-231. https://doi.org/10.1016/j.nanoen.2018.08.054

[23] Sekar, P., Santhoshkumar, P., Joe, Y.C., Senthil, C. and Lee, C.W. (2020) 3D-Printed Architecture of Li-Ion Batteries and Its Applications to Smart Wearable Electronic Devices. Applied Materialstoday, 20, Article ID: 100688. https://doi.org/10.1016/j.apmt.2020.100688

[24] Zhao, J., Lin, Y., Wu, J., Nyein, H.Y.Y., Bariya, M., Tai, L.-C., et al. (2019) A Fully Integrated and Self-Powered Smartwatch for Continuous Sweat Glucose Monitoring. ACS Sensors, 4, 1925-1933. https://doi.org/10.1021/acssensors.9b00891

[25] Zhu, Y.-H., Yang, X.-Y., Liu, T. and Zhang, X.-B. (2020) Flexible 1D Batteries: Recent Progress and Prospects. Advanced Materials, 32, Article ID: 1901961. https://doi.org/10.1002/adma.201901961

[26] Daemi, N., Ahmadian, A., Mirbagheri, A., Ahmadian, A.H., Saberi, H., Amidi, F., et al. (2015) Planning Screw Insertion Trajectory in Lumbar Spinal Fusion Using PreOperative CT Images. Proceedings of the 201537 th Annual International Conference of the IEEE Engineering in Medicine and Biology Society, Milan, 25-29 August 2015, 3639-3642. https://doi.org/10.1109/EMBC.2015.7319181

[27] Akbar (2017) United States Patent (54) Robotic Guide for Brain Biopsy (71). Parseh Intelligent Surgical System, Tehran.

[28] Hasani bidgoli, J., Ahmadian, A., Sadeghi, M.J., Ahmadian, A., Farahmand, F. and Sarkar, S. (n.d.) Device for Brain Biopsy. US Patent No. 20180132962A1. 
https://patents.google.com/patent/US20180132962A1/en

[29] Zhou, G., Li, F. and Cheng, H.M. (2014) Progress in Flexible Lithium Batteries and Future Prospects. Energy and Environmental Science, 7, 1307-1338.

https://doi.org/10.1039/C3EE43182G

[30] Gaikwad, A.M., Arias, A.C. and Steingart, D.A. (2015) Recent Progress on Printed Flexible Batteries: Mechanical Challenges, Printing Technologies, and Future Prospects. Energy Technology, 3, 305-328. https://doi.org/10.1002/ente.201402182

[31] Wu, Z., Wang, Y., Liu, X., Lv, C., Li, Y., Wei, D., et al. (2019) Carbon-Nanomaterial-Based Flexible Batteries for Wearable Electronics. Advanced Materials, 31, Article ID: 1800716. https://doi.org/10.1002/adma.201800716

[32] Hu, Y. and Sun, X. (2014) Flexible Rechargeable Lithium Ion Batteries: Advances and Challenges in Materials and Process Technologies. Journal of Materials Chemistry $A, 2,10712-10738$. https://doi.org/10.1039/C4TA00716F

[33] Zeng, L., Qiu, L. and Cheng, H.M. (2019) Towards the Practical Use of Flexible Lithium ion Batteries. Energy Storage Materials, 23, 434-438.

[34] Ahmadian, A. (2021) A Review on Recent Selective Laser Printing of Medicines. EasyChair Preprint No. 5327.

[35] Ahmadian, A., Alidoost, M., Shafiee, A. and Akbari, A. (2021) Overview of NanoFiber Fabrication via Elec-tro-Spinning Tailored for Energy Storage Systems. Easy Chair Preprint No. 5126.

[36] Ahmadian, A., Shafiee, A., Aliahmad, N. and Agarwal, M. (2021) Overview of Nano-Fiber Bundles Fabrication via Electrospinning and Morphology Analysis. Preprints 2021, 2021040711. https://doi.org/10.20944/preprints202104.0711.v1 